\section{Efectividad de un programa de rehabilitación autoadministrado en el tratamiento del síndrome de hombro doloroso en atención primaria de salud: un estudio clínico aleatorizado, simple ciego}

\author{
JULIO CONTRERAS ${ }^{1,2}$, RODRIGO LIENDO ${ }^{1,3}$, CRISTÓBAL DÍAZ $^{1, a}$, \\ MARÍA DÍAZ ${ }^{1, \mathrm{~b}}$, MATÍAS OSORIO $^{1, \mathrm{c}}$, RICARDO GUZMÁN $^{1, \mathrm{c}}$, \\ FRANCISCO SOZA ${ }^{3}$, MANUEL BELTRÁN ${ }^{1}$, HÉCTOR PALOMO ${ }^{1}$, \\ CARLOS CÓRDOVA ${ }^{2, \mathrm{~d}}$, DANIEL MANOSALVAS ${ }^{2, \mathrm{~d}}$, JUAN LECAROS ${ }^{2, \mathrm{~d}}$, \\ RAMÓN TORRES ${ }^{2, a}$, GILBERTO GRAU ${ }^{2, a}$, PABLO SILVA ${ }^{2, a}$, \\ CRISTIÁN PARADA ${ }^{2, a}$, DOMINIQUE CIBIE ${ }^{\mathrm{e}}$, \\ RODRIGO MARTÍNEZ ${ }^{\mathrm{f}}$, IVÁN PÉREZ ${ }^{\mathrm{f}}$
}

\section{Effectiveness of a self-administered rehabilitation program for shoulder pain syndrome in primary health care}

Background: Shoulder pain syndrome (SPS) is frequent and management in primary care is precarious, with a high rate of referral without adequate treatment, overloading rehabilitation and orthopedic services. Aim: To assess the effectiveness of a self-administered rehabilitation program in adults with shoulder pain syndrome in primary care. Patients and Methods: A randomized, single-blind clinical trial (evaluators) with an experimental group (self-administered rehabilitation) and a control group (standard physical therapy) was carried out in 271 adult patients aged 18 or older with unilateral shoulder pain lasting more than six weeks and less than three months. The primary outcome was the recovery perceived by the patient. Constant score for function, quality of life using SF-36, simple shoulder test (SST) and the Disabilities of the Arm, Shoulder, and Hand (DASH) score were also calculated at six, 12 and 24 weeks of follow-up. Results: The self-administered rehabilitation program showed an adjusted effectiveness of $51 \%$ at the end of treatment compared to $54 \%$ of the standard physical therapy $(p>0.05)$. No differences in the evolution of the other scores assessed were observed between groups. Conclusions: A self-administered rehabilitation program for painful shoulder was non-inferior than usual physical therapy.

(Rev Med Chile 2018; 146: 959-967)

Key words: Physical Therapy Modalities; Shoulder Impingement Syndrome; Shoulder Pain; Rehabilitation.

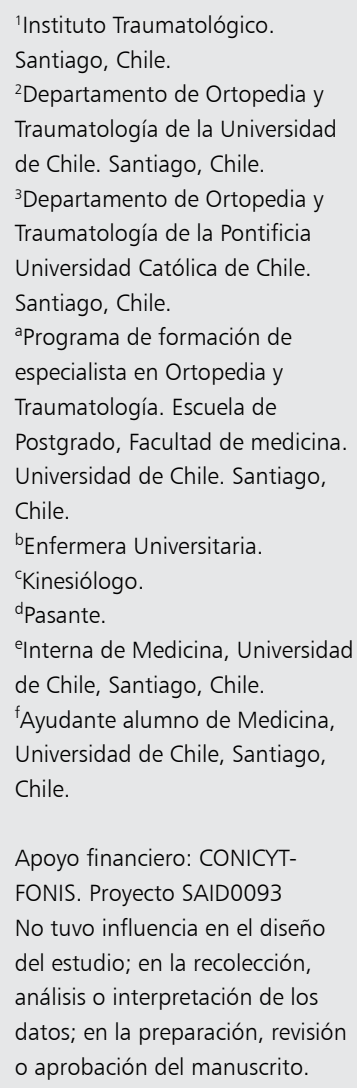

Apoyo financiero: CONICYTFONIS. Proyecto SAID0093 No tuvo influencia en el diseño del estudio; en la recolección, análisis o interpretación de los datos; en la preparación, revisión o aprobación del manuscrito.

Recibido el 25 de febrero de 2018, aceptado el 16 agosto de 2018.

Correspondencia a: Dr. Julio Contreras Fernández Pocuro 2170 Depto 63, Providencia, Santiago, Chile. juliocontrerasmd@gmail.com 
L as enfermedades músculo-esqueléticas (EME) son de alta prevalencia y escaso acceso a la atención, generando listas de espera, insatisfacción usuaria y fracaso clínico ${ }^{1,2}$. El síndrome de hombro doloroso (SHD) es frecuente, representando más de $15 \%$ de todas las consultas por $\mathrm{EME}^{3}$; se calcula una incidencia entre 6,6 y 25 casos por cada 1.000 pacientes $^{4}$, y una prevalencia entre 70 y 260 por 1.000 en población general ${ }^{5-7}$.

En Chile, no se cuenta con estudios epidemiológicos, pero se estima que en un año consultan más de un millón de pacientes. El manejo del SHD en atención primaria (APS) es precario, con alta tasa de derivación sin tratamiento inicial adecuado ni cumplimiento de criterios de derivación, sobrecargando los servicios de rehabilitación y ortopedia a nivel secundario y terciario ${ }^{1}$. Se requieren nuevas estrategias para aumentar la resolutividad y óptima derivación por parte de la APS.

El SHD produce discapacidad, trastornos del sueño, limitaciones en las actividades de la vida diaria (conducir, vestirse, peinarse y comer) y una pérdida de calidad de vida, afectando también la capacidad para trabajar ${ }^{8-11}$. Estos pacientes presentan, además, depresión, ansiedad y trastornos somatomorfos en cuadros con duración mayor a 3 meses $^{12,13}$.

El manejo del SHD es controversial. En España, se detectó un manejo deficiente del SHD en APS. Más de 95\% de los pacientes no cumplía el criterio de tratamiento en los tiempos preestablecidos ${ }^{14}$. El uso temprano de los estudios de imágenes y referencias a especialistas están sobreutilizados por los médicos de APS, aumentando costos y listas de espera ${ }^{15,16}$.

La rehabilitación kinésica es de primera línea. El objetivo de un programa de ejercicios en SHD es aliviar el dolor, aumentar la fuerza, revertir los desequilibrios musculares anormales y restablecer un rango de movimiento articular indoloro ${ }^{17}$. Distintas revisiones sugieren que el ejercicio es eficaz para reducir el dolor y mejorar la función en un período de 6 a 12 semanas ${ }^{17,18}$.

En Reino Unido se han introducido una serie de políticas de salud para hacer frente a la creciente demanda y apoyar el autocuidado. Se está alentando a alejarse de un modelo de dependencia del paciente a uno donde los pacientes se sienten informados y capaces de ejercer control sobre su propia salud (empoderamiento), y, por lo tanto, asumir la responsabilidad de un autotratamiento con la actuación del profesional de la salud como guía y mentor ${ }^{19}$. La profesión kinesiológica tiene una larga historia de promover el uso del autotratamiento entre las consultas; sin embargo, ayudar a los pacientes en el aprendizaje sobre el ejercicio toma mucho tiempo. Los pacientes pueden olvidar sus ejercicios o no llevarlos a cabo entre las consultas ${ }^{20}$.

Una revisión sistemática sugiere que la efectividad de los programas de ejercicio realizados en el hogar pueden ser más eficaces que ninguna intervención o placebo y tan eficaces como órtesis funcionales, kinesioterapia y cirugía ${ }^{21}$. Similarmente, otro metaanálisis encontró que el ejercicio terapéutico tiene un efecto positivo sobre el dolor y la función por encima de todas las demás intervenciones $^{22-24}$.

El objetivo de este estudio fue determinar si en pacientes adultos con SHD atendidos en APS, la aplicación de un programa de rehabilitación autoadministrado (PRAA) comparado con un programa de rehabilitación kinesiológico estándar (PRKE) no es inferior en resultados clínicos, calidad de vida y discapacidad a corto y mediano plazo.

\section{Materiales y Métodos}

\section{Aspectos generales}

Ensayo clínico aleatorizado de no inferioridad, simple ciego (evaluadores): rama experimental (PRAA) y rama control (PRKE); duración total: 36 meses (Santiago, Chile).

\section{Objetivo general}

Establecer la efectividad de un PRAA en pacientes adultos con diagnóstico de SHD que consultan en APS

\section{Objetivos específicos}

1. Describir las características demográficas, socioeconómicas y clínicas en pacientes adultos con SHD que consultan en APS.

2. Analizar la diferencia de efectividad en resultados clínicos, calidad de vida y discapacidad a las 6, 12 y 24 semanas en la aplicación de un PRAA, comparado con un PRKE en pacientes adultos con SHD que consultan en APS. 


\section{Muestra y criterios de inclusión/exclusión}

Bajo técnica de muestreo aleatorio simple, entre mayo de 2015 y julio de 2017, se reclutaron 271 pacientes de APS que consultaban por un SHD pertenecientes a la población inscrita en el Servicio de Salud Metropolitano Occidente. SHD se definió como dolor alrededor del deltoides.

Se incluyeron todos los pacientes adultos con edad igual o mayor a 18 años que consultaron por dolor de hombro unilateral con más de 6 semanas y menos de 3 meses de evolución.

Se excluyeron los pacientes con antecedente traumático, enfermedad inflamatoria articular (artritis reumatoidea, artritis psoriática), tumor óseo, inestabilidad, dolor referido, antecedente de cirugía o rechazo de cirugía, fractura humeral, deterioro psicoorgánico, fibromialgia, embarazo, infiltración o tratamiento kinesiológico dentro de los 6 meses anteriores, trastornos psiquiátricos, neurológicos o vasculares.

Los pacientes que no cumplieron los criterios fueron remitidos a APS con una interconsulta con el tratamiento más adecuado para su patología. Aquellos pacientes que necesitaban evaluación por especialista, fueron ingresados al Policlínico de Hombro del Instituto Traumatológico.

El estudio fue aprobado por el Comité de Ética del Instituto Traumatológico.

\section{Enrolamiento, visita inicial y recolección de información}

Los médicos solicitaban evaluación para los pacientes a través de correo electrónico.

Los pacientes fueron contactados telefónicamente por parte del investigador principal para confirmar los criterios de inclusión y el interés de participar.

Todos los pacientes $(\mathrm{n}=1.237)$ fueron evaluados por el investigador principal (JC) para revisar los criterios de inclusión/exclusión; explicar aspectos generales y objetivos del estudio, cumpliendo $21,9 \%$ (271) de los pacientes derivados. Una vez incluido, se realizó una anamnesis y examen físico por parte de otro evaluador según pauta preestablecida; se completaron los cuestionarios validados para evaluar función (score de Constant-Murley [CS], Simple Shoulder Test $[\mathrm{SST}])^{25}$, calidad de vida $(\mathrm{SF}-36 \mathrm{v} 2)^{26}$ y discapacidad $(\mathrm{DASH})^{27}$; se solicitaron radiografías $\mathrm{y}$ resonancia nuclear magnética; se solicitó y realizó el consentimiento informado; se citaban a control con resultado de exámenes para descartar algún criterio de exclusión imageneológico.

Se completó ficha clínica que contenía preguntas sobre las variables demográficas, socioeconómicas, características clínicas (es decir, la intensidad del dolor, la discapacidad, la duración de las molestias, el inicio y la comorbilidad), la actividad física, la carga de trabajo física y factores psicosociales (Figura 1).

En una segunda evaluación, se revisaron los exámenes, se distribuyó en forma aleatoria al paciente y se entregaban las indicaciones de tratamiento.

La distribución aleatoria se realizó en www. randomization.org, estratificando los pacientes según sexo y edad. El personal del estudio fue ciego a la asignación del tratamiento, solicitando a los participantes no revelar su intervención, en particular durante el examen de seguimiento.

\section{Variables independientes}

- PRAA.

- PRKE.

- Variables modificadoras de efecto (edad, género, comorbilidades, índice de masa corporal, actividad física, trabajo, tiempo de evolución, intensidad de los síntomas, etc.).

\section{Variables dependientes}

Resultado primario

- Recuperación percibida por paciente a las 6 semanas.

\section{Resultados secundarios}

- Recuperación percibida por paciente a las 12 y 24 semanas.

- Estado laboral a las 6, 12 y 24 semanas.

- Cambio en la intensidad del dolor a las 6, 12 y 24 semanas.

- Función asociada a hombro (CS-SST) a las 6, 12 y 24 semanas.

- Calidad de vida (SF-36v2) a las 6, 12 y 24 semanas.

- Discapacidad (DASH) a las 6, 12 y 24 semanas.

\section{Programas de rehabilitación}

Los pacientes PRAA recibieron 1 libro instructivo para la terapia física en el hogar, escrito para un nivel educacional básico con un DVD para reforzar (a todos los pacientes se les reprodujo el video y se les explicó, respondiendo dudas y 
asegurando conceptos claves). Este programa está basado en una revisión sistemática que demostró la eficacia del ejercicio en SHD. Los ejercicios incluyen rango diario de movimiento (ejercicios posturales, movilización activa asistida, entrenamiento activo de los músculos escapulares, rango activo de movimiento); flexibilidad diaria (estiramiento anterior y posterior del hombro); y fortalecimiento muscular 3 veces por semana (ejercicios escapulares y de manguito rotador). Una hoja de registro y cintas elásticas de color verde fueron entregadas al paciente.

Los kinesiólogos PRKE son pertenecientes a la Unidad de Rehabilitación del Instituto Traumatológico. Se realizaron 10 sesiones por cada 6 semanas de tratamiento (40 sesiones en total, según el estándar internacional y nacional).

Ambos grupos recibieron una pauta progresiva de manejo analgésico única.

\section{Maniobra del estudio y seguimiento}

A las 6 semanas se realizaba el primer control clínico por un evaluador ciego a la terapia realizada.

Si el paciente refería un cese de los síntomas y una recuperación de su estado funcional premórbido ("Éxito"), se citaba para evaluación a las 12 y 24 semanas, suspendiendo el tratamiento en ejecución.

Si el paciente refería una disminución, pero persistencia de los síntomas o afectación de su estado funcional, calidad de vida o discapacidad ("Mejoría"), se continuaba el protocolo en ejecución y se realizaba evaluación a las 12 y 24 semanas.

Si el paciente refería persistencia, sin mejoría de los síntomas o afectación de su estado funcional, calidad de vida o discapacidad ("Sin cambios"), se realizaba una apertura del ciego, ofreciendo a los pacientes una cambio de tratamiento.

En aquellos pacientes sin mejoría luego de las 24 semanas de tratamiento ("Fracaso"), se realizaba una evaluación por especialista para definir conducta a seguir.

\section{Análisis estadístico}

Se realizó ensayo clínico de no inferioridad con intención de tratar, utilizando todos los datos disponibles de todos los participantes asignados al azar. Para estimar los efectos de la intervención en el resultado primario se calculó el riesgo relativo e intervalo de confianza a las 6 semanas y secun- dariamente a 12 y 24 semanas, dependiendo del momento de presentación de la condición éxito/ fracaso.

Considerando como resultado la recuperación percibida por el paciente a las 6 semanas y basándose en la literatura (75\% de efectividad), esperando una diferencia no mayor de $15 \%$ respecto al PRAA (63,75\% de efectividad), con un test a dos colas, un nivel de confianza 95\%, poder estadístico $80 \%$, el tamaño muestral fue de 262 .

Los márgenes de equivalencia, sin tener datos nacionales y considerando la literatura variada, en base a panel de expertos, se considero un margen entre $70 \%\left(\Delta^{+}\right)$y $30 \%\left(\Delta^{-}\right)$de efectividad.

Se utilizó la prueba de chi cuadrado para comparación de proporciones y t-Student con corrección de Welch para comparación de medias. Se consideró significativo los estudios a dos colas con un $\mathrm{p}<0,05$. Todos los análisis se realizaron con STATA12 y GraphPad Prism 7.

\section{Resultados}

\section{Población de estudio y seguimiento}

Doscientos setenta y un pacientes fueron interrogados y examinados físicamente. La Tabla 1 enumera las características basales de los participantes. El 71,21\% cumplió ambos tratamientos en forma completa y se utilizaron para análisis estadístico (28,57\% abandonó PRKE y 27,54\% PRAA). Los abandonos en ambos grupos de tratamiento no presentó diferencias significativas en sus características basales. Luego de completar el tratamiento, $45 \%$ de los pacientes PRRA presentó curación, en cambio $60 \%$ de los tratados con PRKE se curó $(\mathrm{p}<0,0361)$ según “intención de tratar".

\section{Resultado primario}

No se encontraron diferencias significativas a las 6 semanas de tratamiento en la recuperación percibida por el paciente (Tabla 2). El PRAA presentó una efectividad con intervalo de confianza 95\% de 36,7 a 53,3\%, en comparación a PRKE con 51,67 a $68,33 \%$.

\section{Resultados secundarios}

Se encontraron diferencias significativas a las 24 semanas de tratamiento en la recuperación percibida por el paciente, obteniendo una superioridad de tratamiento en el PRKE con 60\% de 
Tabla 1. Características basales de pacientes con dolor en el hombro manejados con el programa de rehabilitación autoadministrada y tratamiento kinesiológico estándar

\begin{tabular}{|c|c|c|c|}
\hline Grupo & $\begin{array}{l}\text { Tratamiento } \\
\text { kinesiológico } \\
\text { estándar }\end{array}$ & $\begin{array}{l}\text { Rehabilitación } \\
\text { auto- } \\
\text { administrada }\end{array}$ & $\mathbf{p}$ \\
\hline Tamaño muestral & 133 & 138 & \\
\hline $\begin{array}{l}\text { Factores demográficos } \\
\text { Edad (años); promedio (DE) } \\
\text { Género: femenino } \\
\text { IMC; promedio (DE) }\end{array}$ & $\begin{array}{c}56,40(11,56) \\
77,89 \% \\
30,19(5,58)\end{array}$ & $\begin{array}{c}55,72(12,36) \\
72,45 \% \\
29,85(6,12)\end{array}$ & $\begin{array}{l}\text { NS } \\
\text { NS } \\
\text { NS }\end{array}$ \\
\hline $\begin{array}{l}\text { Factores comórbidos } \\
\text { Diabetes mellitus } 2 \\
\text { Hipertensión } \\
\text { Dislipidemia } \\
\text { Artrosis } \\
\text { Patología cervical }\end{array}$ & $\begin{array}{c}29,47 \% \\
57,89 \% \\
29,47 \% \\
23,16 \% \\
5,26 \%\end{array}$ & $\begin{array}{l}30,61 \% \\
51,02 \% \\
23,47 \% \\
19,39 \% \\
10,20 \%\end{array}$ & $\begin{array}{l}\text { NS } \\
\text { NS } \\
\text { NS } \\
\text { NS } \\
\text { NS }\end{array}$ \\
\hline $\begin{array}{l}\text { Factores laborales } \\
\text { Antigüedad laboral (meses); promedio (DE) } \\
\text { Licencia médica actual } \\
\text { Jubilación actual } \\
\text { Lesión de hombro en otros compañeros } \\
\text { Puntaje de Encuesta de factores ergonómicos; promedio (DE) }\end{array}$ & $\begin{array}{c}177,34(157,43) \\
6,32 \% \\
8,42 \% \\
13,68 \% \\
4,64(1,46)\end{array}$ & $\begin{array}{c}206,29(180,44) \\
8,25 \% \\
12,24 \% \\
17,35 \% \\
5,02(1,65)\end{array}$ & $\begin{array}{l}\text { NS } \\
\text { NS } \\
\text { NS } \\
\text { NS } \\
\text { NS }\end{array}$ \\
\hline $\begin{array}{l}\text { Factores sociales } \\
\text { Pertenencia a pueblo originario } \\
\text { Capacidad de lectura } \\
\text { Capacidad de escritura } \\
\text { Manejo de segundo idioma } \\
\text { Tabaquismo activo } \\
\text { Alcoholismo activo } \\
\text { Puntaje de Encuesta de Nivel Socioeconómico; promedio (DE) }\end{array}$ & $\begin{array}{c}6 \% \\
94,74 \% \\
93,68 \% \\
4,21 \% \\
34,74 \% \\
25,26 \% \\
27,18(20,10)\end{array}$ & $\begin{array}{c}6,12 \% \\
95,92 \% \\
95,91 \% \\
3,06 \% \\
32,65 \% \\
22,45 \% \\
30,05(19,26)\end{array}$ & $\begin{array}{l}\text { NS } \\
\text { NS } \\
\text { NS } \\
\text { NS } \\
\text { NS } \\
\text { NS } \\
\text { NS }\end{array}$ \\
\hline Factores clínicos & & & \\
\hline $\begin{array}{l}\text { Dolor } \\
\text { Lateralidad derecha } \\
\text { Lateralidad izquierda } \\
\text { Tiempo de evolución (semanas); promedio (DE) } \\
\text { Intensidad del dolor (Escala Visual Análoga); promedio (DE) } \\
\text { Dolor nocturno } \\
\text { Episodios de despertar por dolor } \\
\text { Posibilidad de dormir sobre hombro afectado }\end{array}$ & $\begin{array}{c}62,11 \% \\
34,74 \% \\
9,64(5,33) \\
7,61(1,76) \\
88,42 \% \\
84,21 \% \\
26,32 \%\end{array}$ & $\begin{array}{c}58,16 \% \\
40,82 \% \\
10,61(7,73) \\
7,17(1,84) \\
88,78 \% \\
82,65 \% \\
23,47 \%\end{array}$ & $\begin{array}{l}\text { NS } \\
\text { NS } \\
\text { NS } \\
\text { NS } \\
\text { NS } \\
\text { NS } \\
\text { NS }\end{array}$ \\
\hline $\begin{array}{l}\text { Tipo de dolor } \\
\text { Urente } \\
\text { Eléctrico } \\
\text { Punzante } \\
\text { Pesado } \\
\text { Sordo }\end{array}$ & $\begin{array}{c}18,95 \% \\
8,42 \% \\
26,32 \% \\
17,89 \% \\
32,63 \%\end{array}$ & $\begin{array}{c}19,39 \% \\
7,14 \% \\
23,47 \% \\
17,35 \% \\
37,76 \%\end{array}$ & $\begin{array}{l}\text { NS } \\
\text { NS } \\
\text { NS } \\
\text { NS } \\
\text { NS }\end{array}$ \\
\hline $\begin{array}{l}\text { Localización del dolor (Figura 1) } \\
\text { A1 } \\
\text { A2 } \\
\text { A3 } \\
\text { A4 } \\
\text { A5 } \\
\text { A6 }\end{array}$ & $\begin{array}{c}38,95 \% \\
83,16 \% \\
67,37 \% \\
34,74 \% \\
4,21 \% \\
2,11 \%\end{array}$ & $\begin{array}{c}43,88 \% \\
87,76 \% \\
76,53 \% \\
45,92 \% \\
18,37 \% \\
9,18 \%\end{array}$ & $\begin{array}{l}\text { NS } \\
\text { NS } \\
\text { NS } \\
\text { NS } \\
0,002 \\
0,034\end{array}$ \\
\hline
\end{tabular}


Tabla 1. Características basales de pacientes con dolor en el hombro manejados con el programa de rehabilitación autoadministrada y tratamiento kinesiológico estándar (continuación)

\begin{tabular}{|c|c|c|c|}
\hline Grupo & $\begin{array}{c}\text { Tratamiento } \\
\text { kinesiológico } \\
\text { estándar }\end{array}$ & $\begin{array}{c}\text { Rehabilitación } \\
\text { auto- } \\
\text { administrada }\end{array}$ & $\mathbf{p}$ \\
\hline A7 & $2,11 \%$ & $7,14 \%$ & NS \\
\hline A8 & $3,16 \%$ & $7,14 \%$ & NS \\
\hline A9 & $12,63 \%$ & $11,22 \%$ & NS \\
\hline A10 & $10,53 \%$ & $11,22 \%$ & NS \\
\hline P1 & $25,26 \%$ & $29,59 \%$ & NS \\
\hline P2 & $47,37 \%$ & $56,12 \%$ & NS \\
\hline P3 & $31,58 \%$ & $46,94 \%$ & NS \\
\hline P4 & $13,68 \%$ & $29,59 \%$ & NS \\
\hline P5 & $7,37 \%$ & $11,22 \%$ & NS \\
\hline P6 & $0,00 \%$ & $6,12 \%$ & 0,0143 \\
\hline P7 & $0,00 \%$ & $3,06 \%$ & NS \\
\hline \multicolumn{4}{|l|}{ Dolor a la palpación } \\
\hline Escotadura esternal & $12,63 \%$ & $9,18 \%$ & NS \\
\hline Esternoclavicular & $14,74 \%$ & $12,24 \%$ & NS \\
\hline Clavícula & $28,42 \%$ & $22,45 \%$ & NS \\
\hline Coracoides & $47,37 \%$ & $52,04 \%$ & NS \\
\hline Acromioclavicular & $46,32 \%$ & $50,00 \%$ & NS \\
\hline Acromion & $55,79 \%$ & $46,94 \%$ & NS \\
\hline Surco bicipital & $68,42 \%$ & $63,27 \%$ & NS \\
\hline Espina escapular & $40,00 \%$ & $35,71 \%$ & NS \\
\hline Subacromial & $70,52 \%$ & $65,31 \%$ & NS \\
\hline Axila y surco bicipital proximal & $31,58 \%$ & $28,57 \%$ & NS \\
\hline \multicolumn{4}{|l|}{ Rangos de movimiento } \\
\hline Elevación anterior pasiva (grados); promedio (DE) & $157,8(32,25)$ & $163,5(23,29)$ & NS \\
\hline Elevación anterior activa (grados); promedio (DE) & $147,4(40,78)$ & $151,5(33,97)$ & NS \\
\hline Abducción pasiva (grados); promedio (DE) & $149,5(38,27)$ & $154,9(32,02)$ & NS \\
\hline Abducción activa (grados); promedio (DE) & $138,8(42,79)$ & $145,7(39,61)$ & NS \\
\hline Aducción pasiva (grados); promedio (DE) & $38,78(19,62)$ & $37,21(14,32)$ & NS \\
\hline Aducción activa (grados); promedio (DE) & $37,42(19,5)$ & $36,95(19,00)$ & NS \\
\hline Rotación interna clínica (grados); promedio (DE) & $7,66(2,61)$ & $7,65(2,50)$ & NS \\
\hline Rotación externa pasiva (grados); promedio (DE) & $57,55(25,10)$ & $50(21,77)$ & 0,0277 \\
\hline Rotación externa activa (grados); promedio (DE) & $52,18(24,27)$ & $46,94(20,95)$ & NS \\
\hline Extensión pasiva (grados); promedio (DE) & $38,05(14,67)$ & $37,19(13,66)$ & NS \\
\hline Extensión activa (grados); promedio (DE) & $36,11(14,33)$ & $35,36(12,73)$ & NS \\
\hline \multicolumn{4}{|l|}{ Pruebas especiales } \\
\hline Test de Neer & $80,00 \%$ & $68,37 \%$ & NS \\
\hline Test de Hawkins & $78,95 \%$ & $70,41 \%$ & NS \\
\hline Test de Jobe & $71 \%$ & $67,35 \%$ & NS \\
\hline Test de Patte & $51,58 \%$ & $52,04 \%$ & NS \\
\hline Test de "Belly - Press" & $27,37 \%$ & $30,61 \%$ & NS \\
\hline Test de "Bear - Hug" & $27,37 \%$ & $30,61 \%$ & NS \\
\hline Test de "Drop - Arm" & $4,21 \%$ & $1,02 \%$ & NS \\
\hline Test de "Horn-Blower" & $6,32 \%$ & $1,02 \%$ & NS \\
\hline Test de Speed & $71,58 \%$ & $69,39 \%$ & NS \\
\hline Test de Yergason & $42,11 \%$ & $48,98 \%$ & NS \\
\hline Test de O'brien & $15,79 \%$ & $19,39 \%$ & NS \\
\hline Test de "Cross-Arm" & $18,95 \%$ & $28,57 \%$ & NS \\
\hline
\end{tabular}

DE: Desviación estándar, NS: No significativo, IMC: Índice de masa corporal. 
Tabla 2. Resultados de curación, dolor, función, calidad de vida y discapacidad según tipo de tratamiento y seguimiento

\begin{tabular}{|c|c|c|c|c|c|c|c|c|}
\hline \multirow[b]{2}{*}{ Tratamiento } & \multicolumn{2}{|c|}{ Basal } & \multicolumn{2}{|c|}{6 semanas } & \multicolumn{2}{|c|}{12 semanas } & \multicolumn{2}{|c|}{24 semanas } \\
\hline & PRAA & PRFE & PRAA & PRFE & PRAA & PRFE & PRAA & PRFE \\
\hline \multirow[t]{2}{*}{ Curación (\%) } & & & 25,0 & 34,7 & 36,0 & 49,5 & $45^{*}$ & $60^{*}$ \\
\hline & \multicolumn{2}{|c|}{ Promedio (DE) } & \multicolumn{2}{|c|}{ Promedio (DE) } & \multicolumn{2}{|c|}{ Promedio (DE) } & \multicolumn{2}{|c|}{ Promedio (DE) } \\
\hline EVA & $7,2 \quad(1,8)$ & $7,4 \quad(2)$ & $5,3 \quad(2,7)$ & $4,6 \quad(2,9)$ & $4,7 \quad(2,9)$ & $43 \quad(3,1)$ & $4,9 \quad(3,4)$ & $4,5 \quad(3,0)$ \\
\hline CS & $63,2(15,2)$ & $60,9(17,2)$ & $70,9(18,5)$ & $71,8(19,1)$ & $71,6(18,3)$ & $68,2(18,9)$ & $68,5(20,7)$ & $67,4(19,5)$ \\
\hline SST & $28,4(25,4)$ & $26,5(21,9)$ & $41,7(29,2)$ & $44,3(30,6)$ & $49,6(33,5)$ & $48,3(32,5)$ & $44,4 \quad(37)$ & $46,9(34,4)$ \\
\hline SF-36 v2 CM & $36,9(11,7)$ & $35,9 \quad(9,1)$ & $39,2 \quad(9,8)$ & $38,9(9,3)$ & $42,3(12,5)$ & $44,1(4,6)$ & $47,1(10,7)$ & $48,2 \quad(6,1)$ \\
\hline SF-36 v2 CF & $36,6 \quad(6,3)$ & $34,1 \quad(3,3)$ & $40,2 \quad(7,4)$ & $39,9(5,7)$ & $43,8(10,1)$ & $42,9(6,3)$ & $46,2 \quad(5,3)$ & $47,8(11,1)$ \\
\hline DASH & $50,3(23,1)$ & $53,8(19,7)$ & $38,8(21,2)$ & $39,8(23)$ & $43,4(26,3)$ & $41,7(26,2)$ & $43,6(28,2)$ & $37,4 \quad(28)$ \\
\hline
\end{tabular}

PRAA: Programa de rehabilitación auto-administrado, PRKE: Programa de rehabilitación kinesiológico estándar, DE: Desviación estándar, EVA: Escala Visual Análoga, CS: Score de Constant-Murley, SST: Simple Shoulder Test, SF-36 v2 CM: Componente mental, CF: Componente físico, DASH: Disabilities of the Arm, Shoulder and Hand (DASH) Score. Sin diferencias significativas en grupo de tratamiento, excepto Curación (\%) a las 24 semanas $p=0,0361$ prueba $\chi^{2}$.

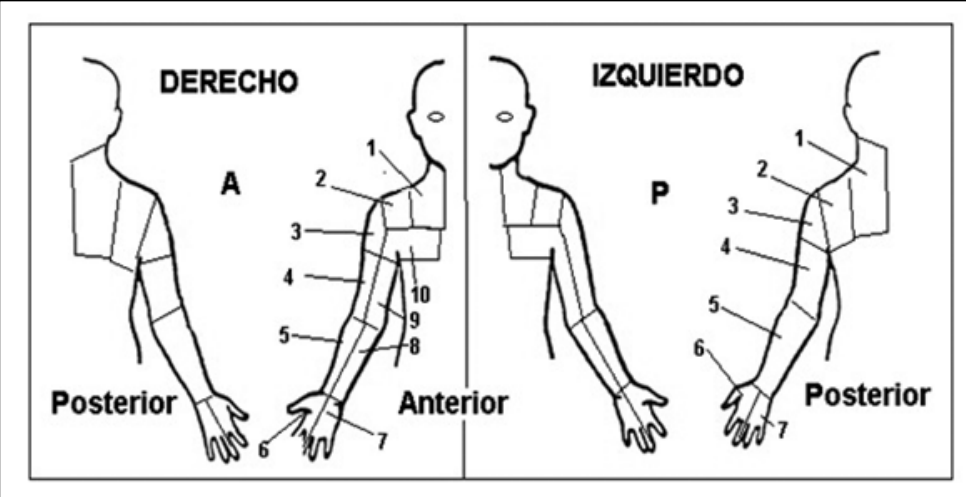

Figura 1.

éxito ( $\mathrm{p}<0,0361)$. Hay que considerar que $21 \%$ de pacientes autoadministrados solicitaron cambio a kinesiología estándar. De estos, 38,1\% logró curación con este cambio. El 19\% de los pacientes de kinesiología estándar solicitó cambio a PRAA. De estos, $61,1 \%$ logró curación con este cambio. Estas diferencias no fueron significativas. Ajustando las tasas de éxito considerando los cambios de grupo de tratamiento, el éxito ajustado del PRAA fue de 51,04\% y del PRKE de 53,54\%. Estas diferencias no fueron significativas.

Al considerar las mediciones objetivas de función, calidad de vida y discapacidad no se encontraron diferencias significativas entre ambos tratamientos en ningún período de seguimiento.
El estado laboral (uso de licencia médica) a las 6,12 y 24 semanas no presentó diferencias significativas entre ambos tratamientos.

\section{Discusión}

La aplicación de un programa de rehabilitación autoadministrado comparado con un programa de rehabilitación kinesiológico estándar en pacientes adultos con SHD atendidos en APS no es inferior en resultados funcionales, calidad de vida y discapacidad a corto y mediano plazo. Respecto a la recuperación percibida por el paciente a corto plazo (6 semanas - 12 semanas) tampoco es in- 
ferior, pero a las 24 semanas hay una percepción de recuperación mayor en aquellos tratados con el PRKE ("intención de tratar"). Considerando los cambios de programa de tratamiento según flujograma, a mediano plazo los resultados serían equivalentes.

La Tabla 1 muestra ambos grupos con sus características basales con mínimas diferencias significativas que impresionan no tener impacto en el análisis de los datos debido a que son diferencias estadísticas pero no clínicamente significativas.

Los resultados son relevantes, ya que la aplicación del PRAA en este subgrupo de pacientes con SHD subagudo no es inferior al manejo kinésico.

En Reino Unido se han introducido una serie de políticas de salud para hacer frente a la creciente demanda y apoyar el autocuidado. Se está impulsando un modelo donde los pacientes se sienten informados y capaces de ejercer control sobre su propia salud ${ }^{19}$. Llama la atención en nuestra población que al mediano plazo, en modalidad "intención de tratamiento", el PRKE presenta mejores resultados desde el punto de vista de subjetividad del paciente, ya que al evaluar en forma objetiva la recuperación con scores funcionales, calidad de vida y discapacidad ambos tratamientos son igual de efectivos y no presentan diferencias significativas. Impresiona que la población chilena pudiese tener un mayor grado de dependencia de la relación terapeuta-paciente. Veintiuno por ciento de los pacientes autoadministrados solicitaron cambio a kinesiología estándar, logrando solo $38,1 \%$ de curación. Muchos pacientes aludieron como explicación de este cambio la necesidad de un kinesiólogo para guiar la terapia, porque "en la casa no hacen los ejercicios". La causa de este problema pudiese estar asociada a la idiosincrasia de la población estudiada. En cambio, 19\% de los pacientes de kinesiología estándar solicitó cambio a PRAA, logrando $61,1 \%$ de curación. Estas diferencias no son significativas, pero impresiona ser un problema de tamaño de la submuestra analizada, ya que el porcentaje de curación es casi $50 \%$. Estos pacientes dejaban la kinesiología asociado a problemas de transporte, horarios ajustados, necesidad de trabajar y la libertad que genera realizar las terapias en casa. Los pacientes se beneficiaron de un enfoque más activo y autosuficiente, que contrasta con la terapia más pasiva en relación con su carga subjetiva de la enfermedad ${ }^{24}$. Una posible explicación es que una terapia en el propio ambiente es más eficaz en la búsqueda y utilización de estrategias de afrontamiento a la enfermedad.

Un estudio clínico aleatorizado que comparó la efectividad un PRKE y un PRAA en el hogar en roturas atraumáticas del manguito rotador, demostró que dos tercios de los pacientes mejoraron clínicamente, independientemente del grupo de terapia. No hubo diferencias significativas entre los grupos con relación al dolor, rango de movimiento, fuerza, score de Constant-Murley y el índice EQ-5D (calidad de vida relacionada con salud ${ }^{24}$, similar a los hallazgos de nuestro estudio.

Agradecimientos: A CONICYT - FONIS por el financiamiento y apoyo metodológico; a todos los funcionarios del Instituto Traumatológico; a los médicos generales de la atención primaria quienes fueron parte fundamental del reclutamiento de pacientes; $y$ a los familiares de los investigadores que apoyaron en el desarrollo de la investigación.

\section{Referencias}

1. Monasterio A, Soto R, Zitko P, Durán F, Keil N. Necesidad de un Programa de Tratamiento del Dolor Musculoesquelético en la Atención Primaria. Kinesiología 2006; 25 (1): 31-6.

2. Encuesta nacional de calidad de vida. 2015-16. INE, MINSAL.

3. Feleus A, Bierma-Zeinstra SM, Miedema HS, Bernsen RM, Verhaar JA, Koes BW. Incidence of non-traumatic complaints of arm, neck and shoulder in general practice. Man Ther 2008; 13: 426-33.

4. van der Windt DA, Koes BW, de Jong BA, Bouter LM. Shoulder disorders in general practice: Incidence, patient characteristics, and management. Ann Rheum Dis 1995; 54: 959-64.

5. Bjelle A. Epidemiology of shoulder problems. Baillieres Clin Rheumatol 1989; 3 (3): 437-51.

6. Green S, Buchbinder R, Glazier R, Forbes A. Interventions for shoulder pain. Cochrane Database Syst Rev 2000; (2): CD001156.

7. Urwin M, Symmons D, Allison T, Brammah T, Busby $\mathrm{H}$, Roxby M, et al. Estimating the burden of musculoskeletal disorders in the community: the comparative prevalence of symptoms at different anatomical site, and the relation to social deprivation. Ann Rheum Dis 1998; 57 (11): 649-55.

8. Greenberg DL. Evaluation and Treatment of Shoulder Pain. Med Clin North Am 2014; 98 (3): 487-504. 
9. Reilly P, Macleod I, Macfarlane R, Windley J, Emery RJ. Dead men and radiologists don't lie: a review of cadaver and radiologic studies of rotator cuff tear prevalence. Ann R Coll Surg Engl 2006; 88 (2): 116-21.

10. Ostör AJ, Richards CA, Prevost AT, Speed CA, Hazleman BL. Diagnosis and relation to general health of shoulder disorders presenting to primary care. Rheumatology 2005; 44 (6): 800-5.

11. Lock C, Allgar V, Jones K, Marples G, Chandler C, Dawson P. Prevalence of back, neck and shoulder problems in the inner city: implications for the provision of physiotherapy services in primary healthcare. Physiother Res Int 1999; 4 (3): 161-9.

12. Bilberg R, Nørgaard B, Overgaard S, Roessler K. Mental health and quality of life in shoulder pain patients and hip pain patients assessed by patient reported outcome. Int J Ortho Trauma Nurs 2014; 18 (2): 81-8.

13. Cho CH, Jung SW, Park JY, Song KS, Yu KI. Is shoulder pain for three months or longer correlated with depression, anxiety, and sleep disturbance? J Shoulder Elbow Surg 2013; 22 (2): 222-8.

14. Marín-Gómez M, Navarro-Collado MJ, Peiró S, Trenor-Gomis C, Payá-Rubio A, Bernal-Delgado E, et al. La calidad de la atención al hombro doloroso. Audit clínico. Gac Sanit 2006; 20 (2): 116-23.

15. Buchbinder R, Staples MP, Shanahan EM, Roos JF. General practitioner management of shoulder pain in comparison with rheumatologist expectation of care and best evidence: an Australian National Survey. PLoS One 2013; 8 (4): e61243.

16. Glazier RH, Dalby DM, Badley EM, Hawker GA, Bell MJ, Buchbinder R, et al. Management of common musculoskeletal problems: a survey of Ontario primary care physicians. CMAJ 1998; 158 (8): 1037-40.

17. Hanratty CE, McVeigh JG, Kerr DP, Basford JR, Finch $\mathrm{MB}$, Pendleton A, et al. The effectiveness of physiotherapy exercises in subacromial impingement syndrome: a systematic review and meta-analysis. Semin Arthritis Rheum 2012; 42 (3): 297-316.

18. Chen JF, Ginn KA, Herbert RD. Passive mobilization of shoulder region joints plus advice and exercise does not reduce pain and disability more than advice and exercise alone: a randomized trial. Aust J Physiother 2009; 55 (1): 17-23.

19. Tyreman S. The expert patient: outline of UK government paper. Med Health Care Philos 2005; 8 (2): 14951.

20. Miller JS, Litva A, Gabbay M. Motivating patients with shoulder and back pain to self-care: can a videotape of exercise support physiotherapy? Physiotherapy 2009; 95 (1): 29-35.

21. Littlewood C, Ashton J, Chance-Larsen K, May S, Sturrock B. Exercise for rotator cuff tendinopathy: a systematic review. Physiotherapy 2012; 98 (2): 101-9.

22. Marinko LN, Chacko JM, Dalton D, Chacko CC. The effectiveness of therapeutic exercise for painful shoulder conditions: a meta-analysis. J Shoulder Elbow Surg. 2011; 20 (8): 1351-9.

23. Kuhn JE. Exercise in the treatment of rotator cuff impingement: a systematic review and a synthesized evidence-based rehabilitation protocol. J Shoulder Elbow Surg 2009; 18 (1): 138-60.

24. Krischak G, Gebhard F, Reichel H, Friemert B, Schneider F, Fisser $\mathrm{C}$, et al. A prospective randomized controlled trial comparing occupational therapy with home-based exercises in conservative treatment of rotator cuff tears. J Shoulder Elbow Surg 2013; 22 (9): 1173-9.

25. Constant C, Gerber C, Emery R, Søjbjerg J, Gohlke F, Boileau P. A review of the Constant score: modifications and guidelines for its use. J Shoulder Elbow Surg 2008; 17 (2): 355-61.

26. Ware, J. SF-36 Health Survey update. Spine 200; 25 (24): 3130-39.

27. Beaton D, Katz J, Fossel A, Wright J, Tarasuk V, Bombardier C. Measuring the whole or the parts? Validity, reliability, and responsiveness of the Disabilities of the Arm, Shoulder and Hand outcome measure in different regions of the upper extremity. J Hand Ther 2001; 14 (2): 128-46. 